\title{
THE NON-INTERACTING MAGNETIZATION FORMULA FROM THE PENDULUM MOTION OF DIPOLES
}

\author{
S. Nagy \\ Institute of Mechatronics, The University of West Hungary, Gasparich M. u. 18/A, H-8900 Zalaegerszeg, Hungary \\ e-mail: sandor.nagy@skk.nyme.hu
}

\begin{abstract}
Non-interacting magnetization Taylor expansions are calculated in 2D and 3D at high external field according to the vibrational model. In this approach all dipoles are moved as a linear pendulum independent from each other. The temperature is taken into account according to the equipartition theorem, thus all particles have the same kinetic energy: $k_{B} T / 2$ in $2 \mathrm{D}$ and $k_{B} T$ in 3D. The expansions valid only at high external magnetic field due to the linearisation of initial differential equation. On the second part of this paper the Maxwell-Boltzmann distribution is taken into account to determine the probability density function of the angular velocity. The ratio of the particles which has zero angular velocity in 2D is the maximum, in 3D is zero. Therefore the theoretical prediction is in better agreement with the exact formula of magnetization in $2 \mathrm{D}$ than in $3 \mathrm{D}$. In this approach the effect of negative magnetization is able to come into existence in the non-interacting fluidum.
\end{abstract}

Keywords: Langevin function, vibrational model, non-interacting magnetization, linear pendulum, 2D and 3D magnetization, negative magnetization

\section{INTRODUCTION}

\subsection{The Taylor expansions of initial magnetization}

The simplest magnetization formula ignores the influence of dipolar interaction between dipolar particles, take into account only the applied external magnetic field. In this case according to the Boltzmann distribution the average of the cosine value of angle between external field and the dipole is:

$$
\langle\cos \varphi\rangle=\frac{\int \cos \varphi \exp (a \cos \varphi) d \Omega}{\int \exp (a \cos \varphi) d \Omega},
$$

where $\varphi$ is the angle between $H$ external field and $m$ strength dipole moment, $a=m H / k_{B} T$, and $\Omega$ denotes the orientation $\left(k_{B}\right.$ : Boltzmann factor, $T$ : temperature). The phrase ,non-interacting” means the independent magnetization divided by saturated magnetization. In $3 \mathrm{D}$ this expression leads to the Langevin function [1]:

$$
\langle\cos \varphi\rangle_{3 D}=\mathcal{L}(a)=\operatorname{coth} a-1 / a .
$$

In 2D the (1) can be written by dividing two Bessel functions [2]:

$$
\langle\cos \varphi\rangle_{2 D}=I_{1}(a) / I_{0}(a),
$$

where $I_{1}$ is the modified Bessel function of the first kind of first order and $I_{0}$ is the modified Bessel function of the first kind of zero order. Performing the Taylor expansion of exponential function in the numerator and denominator of (1) centered at zero and executing the integrals (or performing the expansion of Bessel functions in case of 2D) the well-known expressions of the non-interacting magnetization can be written as: 


$$
\begin{gathered}
\langle\cos \varphi\rangle_{2 D}^{H \rightarrow 0}=\frac{\frac{1}{2} a+\frac{1}{16} a^{3}+\frac{1}{384} a^{5}+\frac{1}{18432} a^{7}+\frac{1}{1474560} a^{9}+\cdots}{1+\frac{1}{4} a^{2}+\frac{1}{64} a^{4}+\frac{1}{2304} a^{6}+\frac{1}{147456} a^{8}+\cdots}=\frac{1}{2} a-\frac{1}{16} a^{3}+\frac{1}{96} a^{5}-\frac{11}{6144} a^{7}+\frac{19}{61440} a^{9}-\cdots \\
\langle\cos \varphi\rangle_{3 D}^{H \rightarrow 0}=\frac{\frac{1}{1.5} a+\frac{1}{15} a^{3}+\frac{1}{420} a^{5}+\frac{1}{22680} a^{7}+\frac{1}{1995840} a^{9}+\cdots}{\frac{1}{0.5}+\frac{1}{3} a^{2}+\frac{1}{60} a^{4}+\frac{1}{2520} a^{6}+\frac{1}{181440} a^{8}+\cdots}=\frac{1}{3} a-\frac{1}{45} a^{3}+\frac{2}{945} a^{5}-\frac{1}{4725} a^{7}+\frac{2}{93555} a^{9}-\cdots
\end{gathered}
$$

These expressions are rewritable into iterational formula:

$$
\begin{gathered}
\langle\cos \varphi\rangle_{2 D}^{H \rightarrow 0}=\frac{\sum_{n=0}^{\infty} \frac{a^{2 n+1}}{X_{n}}}{\sum_{n=0}^{\infty} \frac{a^{2 n}}{Y_{n}}}, \text { where } \begin{array}{r}
X_{0}=2 \\
Y_{0}=1
\end{array}, \text { and } \begin{array}{l}
X_{n}=X_{n-1}\left(4 n^{2}+4 n\right) \\
Y_{n}=Y_{n-1}\left(4 n^{2}\right)
\end{array} \\
\langle\cos \varphi\rangle_{3 D}^{H \rightarrow 0}=\frac{\sum_{n=0}^{\infty} \frac{a^{2 n+1}}{X_{n}}}{\sum_{n=0}^{\infty} \frac{a^{2 n}}{Y_{n}}}, \text { where } \begin{array}{r}
X_{0}=1.5 \\
Y_{0}=0.5
\end{array}, \text { and } \begin{array}{r}
X_{n}=X_{n-1}\left(4 n^{2}+6 n\right) \\
Y_{n}=Y_{n-1}\left(4 n^{2}+2 n\right)
\end{array}
\end{gathered}
$$

moreover dividing by Taylor expansions:

$$
\langle\cos \varphi\rangle_{2 D}^{H \rightarrow 0}=\frac{\sum_{n=0}^{\infty} \frac{a^{2 n+1}}{2^{2 n+1}(n+1) ! n !}}{\sum_{n=0}^{\infty} \frac{a^{2 n}}{2^{2 n} n ! n !}} \quad \text { and } \quad\langle\cos \varphi\rangle_{3 D}^{H \rightarrow 0}=\frac{\sum_{n=0}^{\infty} \frac{2 a^{2 n+1}}{(2 n+1) !(2 n+3)}}{\sum_{n=0}^{\infty} \frac{2 a^{2 n}}{(2 n+1) !}}
$$

At high external field in (2) the $\operatorname{coth}(a)$ tends to 1 and performing the Taylor expansion in (3) when $a$ tends to infinity the magnetization formulas lead to [3]:

$$
\langle\cos \varphi\rangle_{2 D}^{H \rightarrow \infty}=1-\frac{1}{2 a}-\frac{1}{8 a^{2}}-\frac{1}{8 a^{3}}-\frac{25}{128 a^{4}}-\cdots \quad \text { and } \quad\langle\cos \varphi\rangle_{3 D}^{H \rightarrow \infty}=1-\frac{1}{a} .
$$

\subsection{Dipole as a linear pendulum}

The equation of the magnetic dipole motion put into a static magnetic field is $[4,5]$ :

$$
\Theta \ddot{\varphi}+m H \sin \varphi=0,
$$

where $\Theta$ is the moment of inertia calculated to the axis of symmetry of the dipole. After linearising this equation the natural frequency of the undamped, unexcited vibrational system is:

$$
\omega_{0}=\sqrt{m H / \Theta} .
$$

This natural frequency applies to the real vibrational motion when the $\varphi_{0}$ amlitude is smaller than approximately $1^{\circ}$. Without linearising (10) the expressions $\varphi(t)$ and $\dot{\varphi}(t)$ are pretty complicated $[4,5]$. 


\subsection{The Maxwell-Boltzmann distribution to the angular velocity}

The rotational energy of particles follows the Maxwell-Boltzmann distribution [6]. In 2D the rotational degree of freedom of a dipole is one, in $3 \mathrm{D}$ it is two, hence the probability density functions of the angular velocity are:

$$
f_{\dot{\varphi}}=\frac{c}{Z} \exp \left(-\frac{N^{2}}{2 \Theta k_{B} T}\right) \quad(2 \mathrm{D}) \quad \text { and } \quad f_{\dot{\varphi}}=\frac{c}{Z} \exp \left(-\frac{N_{\chi}^{2}+N_{y}^{2}}{2 \Theta k_{B} T}\right), \quad \text { (3D) }
$$

where $Z$ is the partition function, $c$ is a normalizing constant, and $N$ signals the angular momentum. Performing the integrals and transformations, density functions of the angular velocity can be written as

$$
f_{\dot{\varphi}} d \dot{\varphi}=\left(\frac{2 \Theta}{\pi k_{B} T}\right)^{1 / 2} \exp \left(-\frac{\Theta \dot{\varphi}^{2}}{2 k_{B} T}\right) d \dot{\varphi} \quad \text { (2D) } \quad \text { and } \quad f_{\dot{\varphi}} d \dot{\varphi}=\frac{\Theta}{k_{B} T} \dot{\varphi} \exp \left(-\frac{\Theta \dot{\varphi}^{2}}{2 k_{B} T}\right) d \dot{\varphi}
$$

The maximum of the function in $2 \mathrm{D}$ is at zero, while in $3 \mathrm{D}$ the value is zero at zero (see Fig. 1).

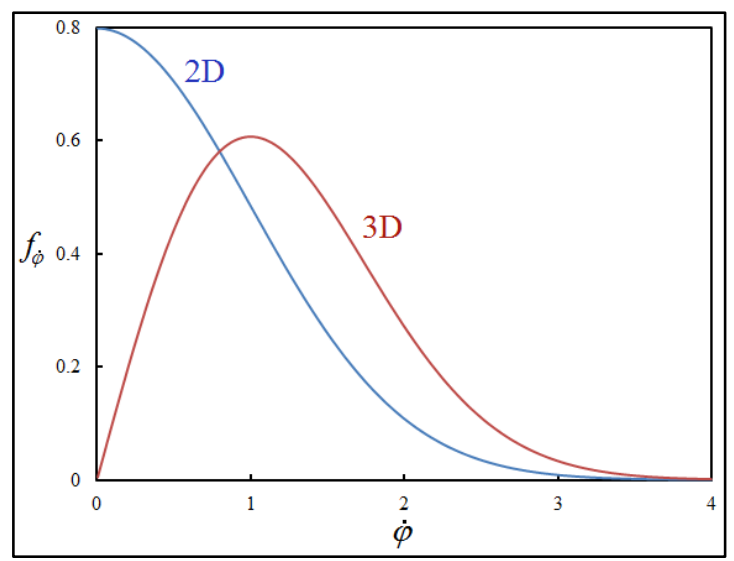

Figure 1. The curves of Maxwell-Boltzmann distribution of angular velocity distribution when the degree of freedom in $2 D$ is one, in $3 D$ is two $\left(\Theta / k_{B} T=1\right)$

\section{MAGNETIZATION FROM PENDULUM MOTION}

According to the equipartition theorem, the average kinetic energy of a particle per degree of freedom is $k_{B} T / 2$. Taking into account the number of the rotational degree of freedom in $2 \mathrm{D}$ and $3 \mathrm{D}$, respectively, this yields:

$$
\frac{1}{2} \theta\left\langle\dot{\varphi}^{2}\right\rangle=\frac{1}{2} k_{B} T \quad \text { (2D) } \quad \text { and } \quad \frac{1}{2} \theta\left\langle\dot{\varphi}^{2}\right\rangle=\frac{2}{2} k_{B} T . \quad \text { (3D) }
$$

Nevertheless, supposing that the dipole is doing harmonic rotational motion [7] taking effect from the external magnetic field, the relation between the average and maximum value of angular velocity is $\left\langle\dot{\varphi}^{2}\right\rangle=\dot{\varphi}_{\max }^{2} / 2$. Using the expression $\dot{\varphi}_{\max }=\varphi_{0} \omega_{0}$ for the amplitude of vibrational motion leads to

$$
\varphi_{0}=\sqrt{\frac{2 k T}{\omega_{0}^{2} \theta}}=\sqrt{\frac{2}{a}}(2 \mathrm{D}) \quad \text { and } \quad \varphi_{0}=\sqrt{\frac{4 k T}{\omega_{0}^{2} \theta}}=\sqrt{\frac{4}{a}} .
$$


It is worth to note that the same results are got to take notice as a starting point of the calculation the vibration energy instead of kinetic energy. In this way

$$
\frac{1}{2} s_{t}\left\langle\varphi^{2}\right\rangle=\frac{1}{2} k_{B} T \quad \text { (2D) } \quad \text { and } \quad \frac{1}{2} s_{t}\left\langle\varphi^{2}\right\rangle=\frac{2}{2} k_{B} T,
$$

where $s_{t}=m H$ is the torsion spring rate and the relation between the average angular displacement value and aplitude is $\left\langle\varphi^{2}\right\rangle=\varphi_{0}^{2} / 2$. Consequently the dipole has a motion around the equilibrium position with $\varphi_{0}$ amplitude and $\omega_{0}$ natural frequency (equilibrium position means that the angle between dipole and external field is zero): $\varphi(t)=\varphi_{0} \sin \left(\omega_{0} t\right)$. The period of oscillation is $T_{p}=2 \pi / \omega_{0}$. The non-interacting magnetization value from the timing average of the external field directional component of dipole moment:

$$
\langle\cos \varphi\rangle=\frac{1}{T_{p}} \int_{0}^{T_{p}} \cos \left(\varphi_{0} \sin \left(\omega_{0} t\right)\right) d t
$$

Performed the expansion of cosine function appear only even powers of sinus function. It is known that

$$
\sin ^{n} x=\frac{1}{2^{n}}\left(\begin{array}{l}
n \\
\frac{n}{2}
\end{array}\right)+\frac{2}{2^{n}} \sum_{k=0}^{\frac{n}{2}-1}(-1)^{\left(\frac{n}{2}-k\right)}\left(\begin{array}{l}
n \\
k
\end{array}\right) \cos ((n-2 k) x),
$$

where $n$ is an even number. The second term of this expression vanishes performing the integral (17) to the whole period. Thus the following simple formula is obtained for the average angular displacement, which is valid for both $2 \mathrm{D}$ and $3 \mathrm{D}$ :

$$
\langle\cos \varphi\rangle=\sum_{n=0}^{\infty}(-1)^{n} \frac{\varphi_{0}^{2 n}}{2^{2 n} n !^{2}}
$$

and using (15) the expansions of non-interacting magnetization function are:

$$
\begin{gathered}
\langle\cos \varphi\rangle_{2 D}=\sum_{n=0}^{\infty}(-1)^{n} \frac{1}{(2 a)^{n} n !^{2}}=1-\frac{1}{2 a}+\frac{1}{16 a^{2}}-\frac{1}{288 a^{3}}+\cdots, \\
\langle\cos \varphi\rangle_{3 D}=\sum_{n=0}^{\infty}(-1)^{n} \frac{1}{a^{n} n !^{2}}=1-\frac{1}{a}+\frac{1}{4 a^{2}}-\frac{1}{36 a^{3}}+\cdots .
\end{gathered}
$$

The first two terms equal to the first two terms in (9), thus one can say that at high $m H / k_{B} T$, where the linearising of (10) is valid, (20) and (21) reproduce the results of the basic theory.

\section{THE NEGATIVE MAGNETIZATION}

According to (13) the Maxwell-Boltzmann distribution is able to apply to the angular velocity of dipoles. The angular velocity on $x$ axes on Fig. 1 corresponds to the time average of angular velocity: $\left\langle\dot{\varphi}_{i}\right\rangle$. The amplitude which belongs to a given $\left\langle\dot{\varphi}_{i}\right\rangle$ average angular velocity:

$$
\varphi_{0 i}=\dot{\varphi}_{i, \max } / \omega_{0}
$$


The average angular velocity is different according to Fig. 1 but the natural frequency and the period is the same for all dipoles at a given temperature. The relation between maximum and average angular velocity referring to a dipole which has a given $\left\langle\dot{\varphi}_{i}\right\rangle$ average angular velocity is:

$$
\left\langle\dot{\varphi}_{i}\right\rangle=\frac{2}{\pi} \dot{\varphi}_{i, \max } .
$$

Consequently the amplitude:

$$
\varphi_{0 i}=\frac{\pi}{2}\left\langle\dot{\varphi}_{i}\right\rangle / \omega_{0}
$$

Using this expression in (19) the magnetization denoted by $\left\langle\dot{\varphi}_{i}\right\rangle$ average angular velocity is: $\langle\cos \varphi\rangle_{i}$. On Fig. 2 the values of $\langle\cos \varphi\rangle_{i}$ are shown as a function of $\left\langle\dot{\varphi}_{i}\right\rangle$ at the values of three different $a=m H / k_{B} T$. When $\left\langle\dot{\varphi}_{i}\right\rangle \rightarrow+0$ the average angular displacement $\langle\cos \varphi\rangle_{i} \rightarrow 1$. Increasing the average angular velocity the aplitude increases and the average angular displacement can turn into the negative zone. The dashed lines sign when the amplitude is greater than $\pi$, thus the linearized equation of motion (10) is not valid yet, because in this case the vibrational motion turn into rotational motion with altering angular velocity. Nevertheless the negative magnetization effect appears somewhere the aplitude is greater than $\pi / 2$ and less than $\pi$, and exists when rotational motion occurs. Hence the positive values of the dashed lines on Fig. 2 are necessarily incorrect.

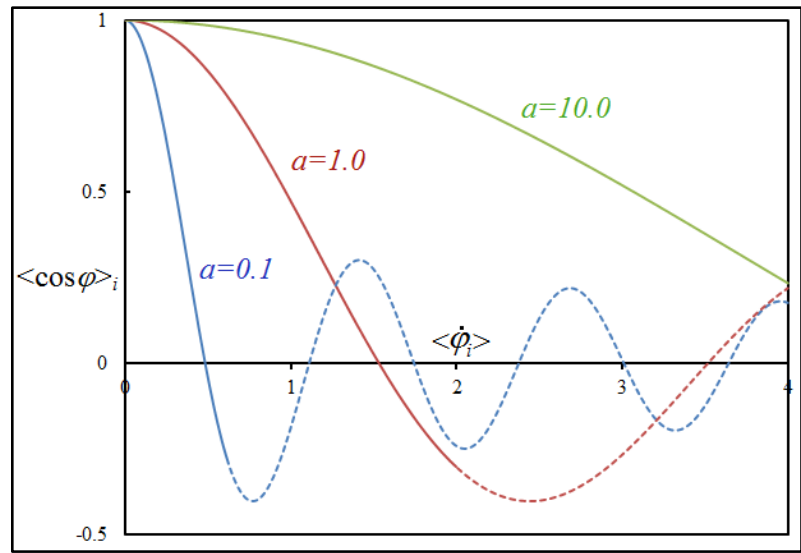

Figure 2. The average angular displacement as a function of the average angular velocity at three different values of $m H / k_{B} T$

The total magnetization from summation part magnetizations weighted by the Maxwell-Boltzmann distribution can be written as (see (13) and (19)):

$$
\langle\cos \varphi\rangle=\int_{0}^{\infty} f_{\dot{\varphi}}\langle\cos \varphi\rangle_{i} d \dot{\varphi} .
$$



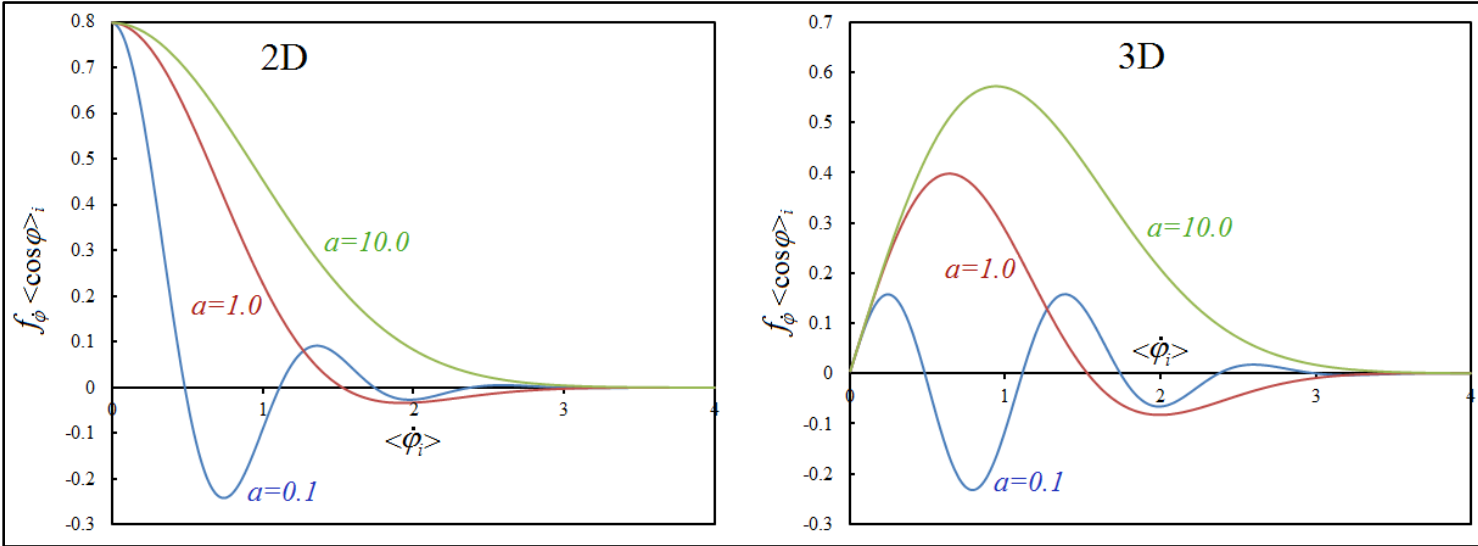

Figure 3. The average angular displacement weighted by the Maxwell-Boltzmann distribution as a function of the average angular velocity at three different values of $\mathrm{mH} / \mathrm{k}_{B} T$. The total magnetization is come from the integration of these functions

On Fig. 3 the curves of part magnetizations are shown in 2D and $3 \mathrm{D}$ at three different values of $a=m H / k_{B} T$. It seems when $a$ is large the all particles have a positive part magnetization. The magnetization of non-interacting fluidum comes from the integration of Maxwell-Boltzmann distribution weighted part magnetizations.

\section{CONCLUSION}

In this paper the Taylor expansion expressions of magnetization of the non-interacting dipoles has been calculated in 2D and 3D on the basis of the vibrational model. The temperature was taken notice with the help of the equipartition theorem which provided the amplitude and the natural frequency of harmonic oscillation. Calculating the average of cosine value of angular excursion a compact formula was arisen for the independent, non-interacting magnetization. Due to the linearising of differential equation, the agreement between our theory and the exact formula is satisfactory only at high external field. Applying the Maxwell-Boltzmann distribution at angular velocity of dipoles, the results have shown that in a noninteracting fluidum exist such particles which have negative magnetization. The main limit of the presented vibrational model is the linearising of (10). In the future an attempt will be made to perform the calculations without linerisation.

\section{REFERENCES}

[1] P. Langevin, Journal de Physique Théorique et Appliquée 4 (1905) 678.

[2] T. Kristóf, I. Szalai, Journal of Physics: Condensed Matter 20 (2008) 204111.

[3] A. O. Ivanov, O. B. Kuznetsova, Journal of Magnetism and Magnetic Materials 252 (2002) 135.

[4] S. V. Kapranov, G. A. Kouzaev, Physica D: Nonlinear Phenomena 252 (2013) 1.

[5] A. Beléndez, C. Pascual, D. I. Méndez, T. Beléndez, C Neipp, Revista Brasileira de Ensino de Física 29 (2007) 645.

[6] N. M. Laurendeau, Statistical thermodynamics: fundamentals and applications, Cambridge University Press, 2005.

[7] B. H. Tongue, Principles of Vibration, Oxford University Press, 2001. 